\title{
Noncompliance with Masking as a Coalitional Signal to US Conservatives in a Pandemic
}

\author{
Kaitlyn Boykin ${ }^{1} \cdot$ Mitch Brown ${ }^{2} \cdot$ Alicia L. Macchione ${ }^{1} \cdot$ Kelsey M. Drea ${ }^{1} \cdot$ Donald F. Sacco $^{1}$
}

Received: 22 November 2020 / Revised: 5 February 2021 / Accepted: 9 February 2021 / Published online: 14 March 2021

(c) The Author(s), under exclusive licence to Springer Nature Switzerland AG part of Springer Nature 2021

\begin{abstract}
Humans have evolved perceptual acuity toward environmental cues heuristically associated with communicable disease that elicits an aversion. One heuristic cue that humans utilize to infer contamination threat is ingroup-outgroup status, with prejudices arising toward outgroup members due to potential novel pathogen exposure. The current study sought to investigate how disease responses in the US population have been modulated by the COVID-19 pandemic, given its origins in China, an outgroup population. We predicted that participants expressing heightened perceived vulnerability to disease and greater levels of conservatism would report higher levels of aversion towards targets not wearing a mask, particularly among Asian targets, given the association of COVID-19 with Asian populations. Results indicate that conservative individuals were more comfortable with both Asian and White targets if they were not wearing a mask, particularly male targets. We contextualize these findings by identifying how mask-wearing during the pandemic could be more communicative of one's coalitional affiliation rather than a protective health measure for more conservative persons.
\end{abstract}

Keywords Disease $\cdot$ Evolutionary psychology $\cdot$ Prejudice $\cdot$ Conservatism $\cdot$ COVID-19

Considering that COVID-19 has become a global pandemic, disease transmission has been made especially salient across cultures. In response to this acute disease threat, numerous novel societal norms have been adopted to facilitate mitigation of further pathogen transmission through social distancing measures. One of the more strongly emphasized strategies for reducing the spread of COVID-19, both because of its relative low cost and ease of implementation, has been mask-wearing (Rader et al., 2020). As mask wearing is a protective health measure that reduces the likelihood of spreading infectious diseases, it should be recognized as a sign of safety among conspecifics, specifically among those with already greater perceived vulnerability to disease transmission.

Although these collective efforts to minimize the spread of COVID-19 might serve to foster a cohesive ingroup identity, outgroups might be consequently stigmatized. For example, based on evidence indicating that COVID-19 likely originated

Kaitlyn Boykin

Kaitlyn.Boykin@usm.edu

1 School of Psychology, The University of Southern Mississippi, 118 College Dr, MS 39406 Hattiesburg, USA

2 University of Arkansas, 1 University of Arkansas, AR 72701 Fayetteville, USA in China (Guo et al., 2020; Shereen et al., 2020), others began implicating Chinese people as responsible for Americans' exposure to this novel pathogenic threat, and subsequently, espoused greater prejudice toward them (Goh, 2020). Group membership is one salient cue humans historically utilize to determine the extent to which a conspecific is a potential pathogenic threat, as outgroups could have introduced novel exogenous pathogens for which one's biological immune system had no preparation (Diamond, 1999). This inference of pathogenic threat posed by outgroups is further heightened when acute pathogen concerns are salient (Faulkner et al., 2004). The current study sought to understand the potentially heightened prejudices formed during the COVID-19 pandemic because of a heightened behavioral immune system response, as indexed by perceived vulnerability to disease, and coalitional motivations to avoid intergroup infections, as indexed by conservative political ideology in the USA.

\section{Pathogen Avoidance and Prejudice}

Despite the evolution of a sophisticated biological immune system that efficiently mitigates the effects of pathogenic infection, such biological responses are nonetheless 
metabolically costly. For example, raising one's body temperature through fever requires a substantial expenditure of metabolic resources (Baracos et al., 1987), thus diverting resources away from other critical fitnessenhancing systems and behaviors (e.g., eating, mating). Individuals would have been at a selective advantage to the extent they could identify and avoid potentially pathogenic conspecifics prior to direct exposure before needing to enact the biological immune system response. It has since been demonstrated that humans evolved a concomitant behavioral immune systems (BIS) that facilitates identification and avoidance of environmental stimuli, as well as conspecifics capable of transmitting infectious disease (Murray \& Schaller, 2016). Salience of disease threats fosters greater interpersonal reticence to reduce contact with prospective disease vectors (Brown \& Sacco, 2016; Mortensen et al., 2010).

Such responses operate along overgeneralization principles of disease threat, wherein individuals overperceive potential disease threats in their environment (Haselton \& Nettle, 2006). Physical features deemed anomalous, albeit non-infectious (e.g., obesity), are particularly aversive to those chronically averse to disease threats (Park et al., 2007). One potential anomalous appearance that could have historically shaped this aversion could include being part of a racial outgroup, with racial prejudice potentially emerging as a consequence of concerns for virulent novel pathogens being transmitted within one's ingroup (see Faulkner et al., 2004; Navarrete \& Fessler, 2006). Within the context of COVID-19 originating in China, it could be possible that Americans utilize Asian facial features as a heuristic cue of pathogen threat in social interactions during the current pandemic. Pathogen-avoidant individuals have indeed given support to travel bans from Asian countries following the outbreak (Goh, 2020), which suggests that proximity with Asian individuals could elicit greater aversion from Americans in the context of the current pandemic. Such findings mirror those of increased reported prejudice toward West African persons during the 2014-2016 Ebola outbreak (Kim et al., 2016).

\section{Coalitional Affiliation}

Within ancestral environments, individuals frequently formed alliances with conspecifics, or coalitions, to facilitate groups' stable access to finite resources by increasing their ability to extract, protect, and secure additional resources through intergroup conflict (Bowles, 2009). Recent work has since argued coalition members who were particularly effective in intergroup conflict subsequently favored the enactment of group policies that favored aggressive social bargaining, which appears to be an ancestral basis for what is deemed conservatism in modern contexts (e.g., Petersen \& Laustsen, 2019; Sinn $\&$ Hayes, 2018). Enactment of these aggressive social policies could further serve to reduce concerns of various outgroup threats, which could include pathogenic threats (Shook et al., 2017). Highly conservative individuals are particularly vigilant toward pathogenic threats (Terrizzi et al., 2013), which ostensibly becomes the impetus of their enactment of policies that restrict intergroup contact and fosters intolerance toward those who may violate local customs (e.g., immigration) in multiple countries (Brown et al., 2019; Brenner \& Inbar, 2015).

Embedded within these conservative coalitions' social policies are additional interests in social binding between ingroup members. Specifically, conservative individuals valuate binding principles of morality that ensure group cohesion and reduces the likelihood of social deviance that may threaten the group's inclusive fitness (Graham et al., 2009). Such binding principles are further emphasized in pathogenic environments, resulting in the increased derogation of non-normative or deviant behaviors (Brown et al., 2017; Thornhill \& Fincher, 2014; Murray et al., 2011) and appearances, including outgroup members (Brenner \& Inbar, 2015). The non-normative behaviors and appearances of outgroup members could represent an increased likelihood of exogenous pathogen threat that would increase the likelihood of novel pathogens within a local ecology for which individuals may not possess a requisite immunocompetence (Diamond, 1999).

These concerns could become particularly exacerbated within a pandemic setting, particularly for groups of people whose ethnic identity has become associated with the pandemic. Given the origins of COVID-19 being in Wuhan, China, individuals report considerable aversion toward Asians when concerns of the pandemic are salient (Goh, 2020; Tabri et al., 2020). Nonetheless, this aversion toward Asians could be ameliorated in the presence of physical cues indicating their interest in reducing disease spread. The Center of Disease Control (CDC) guidelines of wearing face masks during the pandemic could implicate outgroup members as less pathogenically threatening, particularly among those heuristically associating them with the virus. Within collectivistic cultures, wearing face masks demonstrates a courtesy to others to prevent the spread of infections that mirrors the reticent behavior typically valuated in pathogenic ecologies that suggests an interest in facilitating social cohesion (Burgess \& Horii, 2012; Murray \& Schaller, 2012). Given both conservative individuals' valuation of reticence in the service of social cohesion, particularly within the context of a diseased environment, it would seem likely that the presence of face 
masks would reduce the derogation of outgroups associated with a pathogenic threat.

\section{Current Research}

The current study sought to understand how the presence of face masks within the context of COVID-19 could potentially shape interpersonal preferences in the service of reducing one's infection risk. We were particularly interested in the interplay between conservatism and BIS activation through individual differences in perceived vulnerability to disease in a US sample. Given that both individual differences are predictive of a valuation of interpersonal reticence within group contexts (e.g., Brown \& Sacco, 2016; Graham et al., 2009; Murray \& Schaller, 2012), we predicted that conservative and pathogen-avoidant individuals would report greater aversion toward others not wearing masks compared to those wearing masks. Additionally, because of the concomitant wariness toward outgroups, particularly those whose appearance is associated with the spread of COVID-19 (Goh, 2020), we predict that this aversion to unmasked interaction partners would be particularly high for Asian individuals. Data and all supplementary materials for this study are available at https://osf.io/23r4f/?view_ only $=\mathrm{da} 99 \mathrm{fb} 55086 \mathrm{a} 449 \mathrm{bb} 7 \mathrm{ae} 54 \mathrm{ba} 2 \mathrm{~d} 18 \mathrm{a} 694$.

\section{Method}

\section{Participants}

We recruited 351 participants from MTurk in exchange for $\$ 0.50$ (US). Because our statistical model included gender as a variable of interest, we excluded one participant identifying as neither male nor female from the final analyses $\left(n=350 ; 138\right.$ women, $212 \mathrm{men} ; M_{\mathrm{Age}}=37.79$ years, SD $=11.26 ; 56.3 \%$ White, $32.9 \%$ Black, $4 \%$ Asian, $6.3 \%$ Hispanic). A sensitivity analysis indicated that we were adequately powered to detect small effects (Cohen's $f$ $=0.07,1-\beta=0.80$ ). Data were collected in August 2020 during the COVID-19 pandemic and not analyzed until the completion of data collection.

\section{Materials and Procedures}

Target Face Content Participants viewed and evaluated 16 target individuals originating from the Chicago Faces Database that were previously normed to possess equivocal levels of attractiveness (Ma et al., 2015). Faces were of White and Asian men and women (four in each target category) between 18-40 years of age with neutrally expressive faces, which were presented in a random order. Importantly, we utilized two versions of each face, with one being presented without occlusions and the other being altered to wear a standard surgical mask that occluded the nose and mouth. Participants evaluated either masked $(N=$ $165)$ or unmasked targets $(N=185)$ on a between-subject basis to prevent the possibility of demand characteristics influencing their evaluations (Christensen, 2012). See Fig. 1 for sample stimuli.

Interpersonal Perceptions Participants indicated their level of comfortability with each target by responding to six ad hoc items that assessed the extent participants would be comfortable around each target during an active pandemic through various potentially risky scenarios, using a 7-point scale $(1=$ Very Uncomfortable; 7 = Very Comfortable).

Conservatism Participants responded to two questions assessing their political orientation along dimensions of fiscal $(M=4.84$, $\mathrm{SD}=1.66)$ and social issues $(M=4.83, \mathrm{SD}=1.75)$ along 7 -point scales $(1=$ Very Liberal; $7=$ Very Conservative $)$. Scores highly correlated with each other $(r=0.77, p<0.001)$, suggesting that these two items were assessing a singular
Fig 1 Unmasked and masked versions of example female and male stimuli from Asian and White heritage
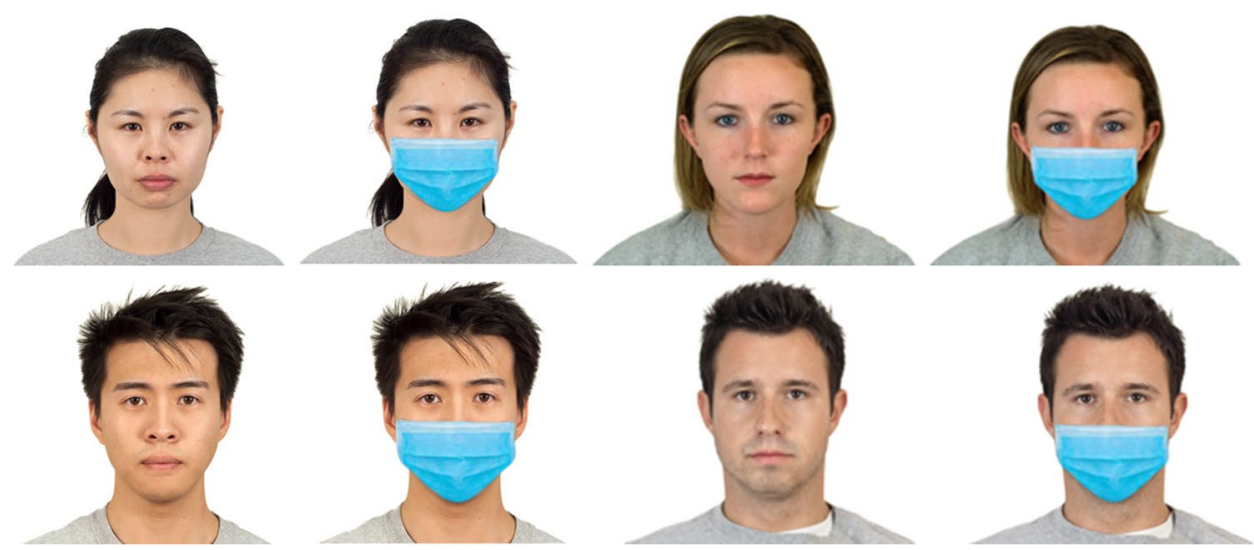
construct at a high degree (i.e., general conservatism). This prompted us to collapse across both orientations to create a composite measure of general conservatism. Separate analyses were, however, conducted for both fiscal and social conservatism on an exploratory basis to facilitate greater comprehension of data. Tables indicating results of these analyses are included as a supplementary file in the OSF link.

Pathogen-Avoidant Motives Participants indicated their dispositional motivation to avoid environmental pathogens using the Perceived Vulnerability to Disease scale (PVD; Duncan et al., 2009). This 15-item measure contains two subscales assessing germ aversion (GA) and perceived infectability (PI) operating along 7-point scales $(1=$ Strongly Disagree; 7 = Strongly Agree). Initial reliability analyses indicated participants responded to the six reverse-scored items as if they were positively keyed ( $\alpha \mathrm{s}$ $<0.55$ ), suggesting a severe acquiescence bias on PVD in the current sample. This prompted us not to consider this scale any further. Bivariate correlations including both GA and $\mathrm{Pi}$ were nonetheless conducted despite these reliability concerns; we provide results of these correlations with study variables in a supplementary file available through the OSF link.

Consenting participants were tasked with evaluating targets before responding to the personality measures. This was followed by demographics and debriefing.

\section{Results}

We conducted a 2 (Participant Sex: Male vs. Female) $\times$ 2 (Target Mask: Masked vs. Unmasked) $\times 2$ (Target Sex: Male vs. Female) $\times 2$ (Target Race: Asian vs. White) mixed-model custom ANCOVAs with repeated factors over the latter two factors using Conservatism as a custom covariate. Entering Conservatism into our model as a custom covariate allows it to serve as a continuous moderator in an omnibus analysis, which affords us to test for interactive effects between continuous predictors and within-subject variables in the same omnibus model (Sacco \& Brown, 2018). Two superordinate interactions emerged, which are from the omnibus model and reported below. These interactions subsequently elicited subordinate interactions that were subsequently decomposed if they were conventionally significant $(\alpha$ $=0.05$ ). Lower-order interactions that were superseded by a subordinate interaction were not reported to deflate the likelihood of Type I Errors.
A Target Race main effect emerged indicating participants were more comfortable around White targets $(M=4.54, \mathrm{SD}$ $=1.51)$ than Asian targets $(M=4.46, \mathrm{SD}=1.52), F(1$, $342)=8.43, p=0.004, \eta_{p}{ }^{2}=0.024$. A Target Sex main effect additionally indicated that participants were more comfortable around female targets $(M=4.74, \mathrm{SD}=1.50)$ than male targets $(M=4.45, \mathrm{SD}=1.54), F(1,342)=12.02$, $p=0.001, \eta_{p}^{2}=0.032$.

\section{Participant and Target Sex Effects}

Effects were initially qualified by a superordinate Participant Sex $\times$ Target Condition $\times$ Target Sex $\times$ Conservatism interaction, $F(1,342)=4.48, p=0.035$, $\eta_{p}{ }^{2}=0.013$. This prompted us to decompose this superordinate interaction by conducting two subordinate 3-way ANCOVAs, which were separate for men and women. No interaction emerged for women, prompting us to consider them no further in this subordinate model, $F(1,134)=0.23, p=0.634, \eta_{p}{ }^{2}=0.002$.

Effects for men were conversely qualified by a subordinate Target Condition $\times$ Target Sex $\times$ Conservatism interaction, $F(1,208)=7.11, p=0.008, \eta_{p}^{2}=0.033$. This prompted us to conduct two subordinate custom Target Sex $\times$ Conservatism ANCOVAs for men's evaluation of masked and unmasked targets separately. The subordinate 2-way interaction was not significant for masked targets and prompted no further consideration, $F(1,96)=1.41, p=$ $0.237, \eta_{p}{ }^{2}=0.015$. Conversely, effects were subordinately qualified by a 2 -way interaction for unmasked targets, $F(1$, $112)=6.20, p=0.014, \eta_{p}{ }^{2}=0.052$. A positive correlation emerged between conservatism and men's comfort in interacting with unmasked men $(r=0.41, p<0.001)$ and women $(r=0.37, p<0.001)$, suggesting that higher levels of conservatism were associated with more comfort around unmasked men and unmasked women.

\section{Target Race Effects}

Effects were further qualified by an additional superordinate Target Mask $\times$ Target Race $\times$ Conservatism interaction, $F(1$, $342)=7.39, p=0.007, \eta_{p}^{2}=0.021$. We decomposed this interaction by conducting two subordinate Target Race $\times$ Conservatism repeated ANCOVAs, separate for masked and unmasked targets. Effects for masked targets were not qualified by a subordinate 2-way interaction and were considered no further, $F(1,163)=0.78, p=0.377, \eta_{p}{ }^{2}=$ 0.005 .

Effects for unmasked targets were qualified by a subordinate Target Race $\times$ Conservatism interaction, $F(1$, 183) $=8.41, p=0.004, \eta_{p}{ }^{2}=0.044$. We decomposed this 
interaction by individually correlating conservatism scores with reported comfort toward unmasked White targets and unmasked Asian targets. A positive correlation emerged between conservatism and comfort with Asian targets, such that higher scores of conservatism were associated with greater comfort around Asian targets not wearing masks ( $r$ $=0.41, p<0.001)$. A similar positive correlation emerged for White targets at a slightly reduced magnitude $(r=0.36, p$ $<0.001)$. No other main effects or superordinate interactions emerged in the omnibus analysis, prompting no further consideration of those effects, $F \mathrm{~s}<1.44, p \mathrm{~s}>0.231$.

\section{Discussion}

Results from the current study provided mixed support for hypotheses. Consonant with previous research, we found that participants indicated more comfort around White targets as opposed to Asian targets, an outgroup stigmatization that is particularly heightened through concerns of disease (Faulkner et al., 2004; Goh, 2020; Kurzban \& Leary, 2001). Additionally, participants indicated greater levels of comfort around female targets as opposed to male targets, with female targets likely being perceived as posing less of an interpersonal threat than male targets. This is consistent with physical size asymmetries imposed by sexual dimorphism, with men being typically larger and stronger (Sell et al., 2012), making men greater interpersonal threats at a base level.

Contrary to original hypotheses, we nonetheless found that conservatism was associated with greater comfort in affiliating with targets without masks. Even more surprisingly, this comfort with unmasked targets was particularly apparent among Asian targets. Though we originally considered mask-wearing as a social rule enforced as a protective health measure, these results offer insight into the possible dual signaling function of masks. If participants did not view wearing a mask as a means of protecting oneself and others from potentially harmful pathogens, mask-wearing might have been viewed as a signal to another's coalition status, given the resistance among certain conservative populations to mask-wearing within the context of the current global pandemic and political climate in the USA (Palmer \& Peterson, 2020). Conservatives may thus view those not wearing a mask as belonging to their ingroup. Additionally, this inclusion might have served as a buffer for Asian targets, who otherwise would have been viewed as outgroup members. Comfort around unmasked individuals could serve to facilitate group cohesion conducive to coalitional success (Fessler \& Holbrook, 2016). Alternatively, these effects could be driven by liberal participants having a greater aversion towards targets without a mask.
Greater acceptance of outgroup members wearing a mask, though surprising, could potentially be a manifestation of the ingroup over-exclusion effect, wherein individuals are normally strict with ingroupoutgroup boundaries (Leyens \& Yzerbyt, 1992; Yzerbyt et al., 1995). It is possible that conservatives were more positive toward Asian targets without masks due to a shared value of individual rights, in this case the right to make a personal choice regarding wearing a mask. This could possibly explain the greater rates of exclusion of both Asian and White targets wearing a mask by conservative participants. As for potential members more ambiguous in their ability to meet the criterion of group acceptance, such as those Asian targets without a mask, more peripheral cues such as wearing versus not wearing a mask might serve as a buffer against the more central cue of belonging to a racial outgroup.

Outside of coalitional affiliation, an alternative explanation could be that participants perceived the presence of a mask as being indicative of disease. Considering the cultural novelty of wearing a mask in the USA, wherein previously wearing a mask would have been predominantly associated with one being sick, these prior perceptions could result in greater aversion among these targets during a pandemic. Conversely, targets not wearing a mask might have been perceived as being healthy, either by virtue of not possessing harmful pathogens or by having strengthened immunity, as they did not find wearing a mask necessary. Although the veracity of these initial perceptions cannot be readily determined by an individual, it could be the case that the available cue of wearing or not wearing a mask serves as the heuristic for interacting versus avoiding a stranger (Haselton \& Nettle, 2006). Furthermore, because these motives cannot be differentiated simply through perception of the target, it could be safer to determine that a person wearing a mask is sick rather than to risk encountering conspecifics already infected with harmful pathogens.

\section{Limitations and Future Directions}

While the current results were able to offer valuable insight into the dual signaling function offered by wearing or not wearing a mask during the current pandemic, there were several notable limitations to the study. One limitation, and likely the most substantial, is that of our PVD results. We were collecting data for this study using an online sample, which could have resulted in participants being less attentive as the study progressed due to its length with PVD being one of the last tasks participants completed. Standard MTurk studies are typically brief; the current study being longer than standard could have contributed to potential study fatigue in the current study. Future studies utilizing online 
paradigms should consider placing attentiveness reminders or employ more attention checks to ensure greater data fidelity that becomes critical with lengthier studies (Kane \& Barabas, 2019; Mancosu et al., 2019).

Future research would additionally benefit from further investigating the relation between political ideology and compliance with COVID-19 transmission mitigation tactics (i.e., wearing a mask). Because our study only included two questions regarding one's political affiliation, our results lacked a level of nuance that could have been gained through including additional items related to participant's espoused conservatism (e.g., Clark et al., 2020). This could also aid in determining whether effects were driven by comfortability among conservative participants or aversion among liberal participants.

An additional follow-up study regarding political ideology could look further into the type of mask worn by targets. For example, perhaps targets wearing masks overtly displaying coalitional status, such as wearing a mask with an American Flag design, would be viewed more favorably than those targets wearing a standard surgical mask. When a common ingroup identity is salient, individuals report less intergroup bias (Gaertner et al., 1993). A mask design emphasizing a superordinate category membership via national identity could facilitate more favorable reactions toward targets to overcome resistance to mask-wearing among certain conservative populations who have greater proclivity to express their patriotism more overtly through symbolic means compared to their partisan counterparts (Huddy \& Khatib, 2007). Lastly, because our sample was comprised of predominantly White participants, future studies would benefit from including a more racially heterogeneous sample to gain a more nuanced look into these intergroup relations. It is possible that inclusion of a larger number of Asian participants would show an increase in comfort among targets shown wearing a mask, as Asian participants might view those wearing a mask as aiming to signal to others their intentions to aid in mitigating the spread of the disease for which Asian populations have been deemed at least partially responsible in initially spreading.

\section{Conclusion}

The current study was able to provide insight as to the possible dual signaling function of wearing a mask versus not wearing a mask during COVID-19, particularly discussing the current study's results in a way that takes a more nuanced look into the value a mask has in signaling one's coalitional status such as political affiliation in the USA based on some unexpected findings. Understanding how wearing a mask might have become politicized during the current pandemic could aid in prompting others to implement these protective health measures to prevent further spread of COVID-19.

Author Contribution Kaitlyn Boykin conceived the study, programmed the study, and wrote the initial draft. Mitch Brown conducted primary analyses and contributed to revisions. Alicia Macchione contributed to revisions. Kelsey Drea contributed to revisions. Donald Sacco assisted in conceiving the study and revisions.

Data Availability https://osf.io/23r4f/?view_only=62dd $45 \mathrm{fd} 35 \mathrm{~cd} 402$ $7948154 \mathrm{a} 0 \mathrm{af} 7587 \mathrm{cc}$

\section{Declarations}

Ethics Approval This project and this consent form have been reviewed by the Institutional Review Board (approval code: IRB-19-284).

Consent to Participate Participants provided informed consent before participating.

Consent for Publication We consent for publication.

Conflict of Interest The authors declare that they have no conflict of interest.

\section{References}

Baracos, V. E., Whitmore, W. T., \& Gale, R. (1987). The metabolic cost of fever. Canadian Journal of Physiology and Pharmacology, $65,1248-1254$.

Bowles, S. (2009). Did warfare among ancestral hunter-gatherers affect the evolution of human social behaviors? Science, 324, 1293-1298.

Brenner, C. J., \& Inbar, Y. (2015). Disgust sensitivity predicts political ideology and policy attitudes in the Netherlands. European Journal of Social Psychology, 45(1), 27-38.

Brown, M., \& Sacco, D. F. (2016). Avoiding extraverts: pathogen concern downregulates preferences for extraverted faces. Evolutionary Psychological Science, 2, 278-286.

Brown, M., Keefer, L. A., Sacco, D. F., \& Bermond, A. (2019). Is the cure a wall? Behavioral immune system responses to a disease metaphor for immigration. Evolutionary Psychological Science, 5, 343-356.

Brown, M., Rodriguez, D. N., Gretak, A. P., \& Berry, M. A. (2017). Preliminary evidence for how the behavioral immune system predicts juror decision-making. Evolutionary Psychological Science, 3, 325-334.

Burgess, A., \& Horii, M. (2012). Risk, ritual and health responsibilisation: Japan's 'safety blanket' of surgical face mask-wearing. Sociology of Health \& Illness, 34(8), 1184-1198.

Christensen, L. (2012). Types of designs using random assignment. In H. Cooper, P. M. Camic, D. L. Long, A. T. Panter, D. Rindskopf, $\&$ K. J. Sher (Eds.). APA handbook of research methods in psychology: Vol. 2. Research designs: Quantitative, qualitative, neuropsychological, and biological (pp. 469-488). American Psychological Association.

Clark, C., Davila, A., Regis, M., \& Kraus, S. (2020). Predictors of COVID-19 voluntary compliance behaviors: an international investigation. Global Transitions, 2, 76-82. 
Diamond, J. (1999). Guns, germs, and steel: The fates of human societies. New York, NY: W. W. Norton.

Duncan, L. A., Schaller, M., \& Park, J. H. (2009). Perceived vulnerability to disease: development and validation of a 15 -item self-report instrument. Personality and Individual Differences, $47,541-546$.

Faulkner, J., Schaller, M., Park, J., \& Duncan, L. (2004). Evolved disease-avoidance mechanisms and contemporary xenophobic attitudes. Group Processes \& Intergroup Relations, 7, 333-353.

Fessler, D. M., \& Holbrook, C. (2016). Synchronized behavior increases assessments of the formidability and cohesion of coalitions. Evolution and Human Behavior, 37, 502-509.

Gaertner, S. L., Dovidio, J. F., Anastasio, P. A., Bachman, B. A., \& Rust, M. C. (1993). The common ingroup identity model: recategorization and the reduction of intergroup bias. European Review of Social Psychology, 4, 1-26.

Goh, J. (2020). Perceived vulnerability to disease predicts restrictive policy supports in response to the 2019-nCoV ("Wuhan virus") outbreak. Preprint on PsyArxiv.

Graham, J., Haidt, J., \& Nosek, B. A. (2009). Liberals and conservatives rely on different sets of moral foundations. Journal of Personality and Social Psychology, 96(5), 1029.

Guo, Y. R., Cao, Q. D., Hong, Z. S., Tan, Y. Y., Chen, S. D., Jin, H. J., $\&$ Yan, Y. (2020). The origin, transmission and clinical therapies on coronavirus disease 2019 (COVID-19) outbreak-an update on the status. Military Medical Research, 7(1), 1-10.

Haselton, M. G., \& Nettle, D. (2006). The paranoid optimist: an integrative evolutionary model of cognitive biases. Personality and Social Psychology Review, 10, 47-66.

Huddy, L., \& Khatib, N. (2007). American patriotism, national identity, and political involvement. American Journal of Political Science, 51(1), 63-77.

Kane, J. V., \& Barabas, J. (2019). No harm in checking: using factual manipulation checks to assess attentiveness in experiments. American Journal of Political Science, 63, 234-249.

Kim, H. S., Sherman, D. K., \& Updegraff, J. A. (2016). Fear of Ebola: The influence of collectivism on xenophobic threat responses. Psychological Science, 27(7), 935-944.

Kurzban, R., \& Leary, M. R. (2001). Evolutionary origins of stigmatization: The functions of social exclusion. Psychological Bulletin, 127(2), 187

Leyens, J.-P., \& Yzerbyt, V. Y. (1992). The ingroup overexclusion effect: impact of valence and confirmation on stereotypical information search. European Journal of Social Psychology, 22, 549-569.

Ma, D. S., Correll, J., \& Wittenbrink, B. (2015). The Chicago face database: a free stimulus set of faces and norming data. Behavior Research Methods, 47, 1122-1135.

Mancosu, M., Ladini, R., \& Vezzoni, C. (2019). 'Short is better'. Evaluating the attentiveness of online respondents through screener questions in a real survey environment. Bulletin of Sociological Methodology, 141, 30-45.

Mortensen, C. R., Becker, D. V., Ackerman, J. M., Neuberg, S. L., \& Kenrick, D. T. (2010). Infection breeds reticence: the effects of disease salience on self-perceptions of personality and behavioral avoidance tendencies. Psychological Science, 21, 440-447.

Murray, D. R., \& Schaller, M. (2012). Threat (s) and conformity deconstructed: Perceived threat of infectious disease and its implications for conformist attitudes and behavior. European Journal of Social Psychology, 42(2), 180-188.
Murray, D. R., \& Schaller, M. (2016). The behavioral immune system: implications for social cognition, social interaction, and social influence. Advances in Experimental Social Psychology, 53, $75-129$.

Murray, D. R., Trudeau, R., \& Schaller, M. (2011). On the origins of cultural differences in conformity: Four tests of the pathogen prevalence hypothesis. Personality and Social Psychology Bulletin, 37(3), 318-329.

Navarrete, C. D., \& Fessler, D. M. T. (2006). Disease avoidance and ethnocentrism: the effects of disease vulnerability and disgust sensitivity on intergroup attitudes. Evolution and Human Behavior, 27, 270-282.

Palmer, C. L., \& Peterson, R. D. (2020). Toxic Mask-ulinity: The link between masculine toughness and affective reactions to mask wearing in the COVID-19 era. Politics \& Gender, 16(4), 1044-1051.

Park, J. H., Schaller, M., \& Crandall, C. S. (2007). Pathogen-avoidance mechanisms and the stigmatization of obese people. Evolution and Human Behavior, 28, 410-414.

Petersen, M. B., \& Laustsen, L. (2019). Upper-body strength and political egalitarianism: Twelve conceptual replications. Political Psychology, 40(2), 375-394.

Rader, B., White, L. F., Burns, M. R., Chen, J., Brilliant, J., Cohen, J., ... \& Astley, C. M. (2020). Mask wearing and control of SARSCoV-2 transmission in the United States. Preprint available on MedRxiv.

Sacco, D., \& Brown, M. (2018). Preferences for facially communicated big five personality traits and their relation to self-reported big five personality. Personality and Individual Differences, 134, 195-200.

Sell, A., Hone, L. S., \& Pound, N. (2012). The importance of physical strength to human males. Human Nature, 23, 30-44.

Shereen, M. A., Khan, S., Kazmi, A., Bashir, N., \& Siddique, R. (2020). COVID-19 infection: origin, transmission, and characteristics of human coronaviruses. Journal of Advanced Research.

Shook, N. J., Ford, C. G., \& Boggs, S. T. (2017). Dangerous worldview: a mediator of the relation between disgust sensitivity and social conservatism. Personality and Individual Differences, 119, 252-261.

Sinn, J. S., \& Hayes, M. W. (2018). Is political conservatism adaptive? Reinterpreting right-wing authoritarianism and social dominance orientation as evolved, sociofunctional strategies. Political Psychology, 39(5), 1123-1139.

Tabri, N., Hollingshead, S., \& Wohl, M. (2020). Framing COVID19 as an existential threat predicts anxious arousal and prejudice towards Chinese people.

Terrizzi, J. A., Jr., Shook, N. J., \& McDaniel, M. A. (2013). The behavioral immune system and social conservatism: a metaanalysis. Evolution and Human Behavior, 34, 99-108.

Thornhill, R., \& Fincher, C. L. (2014). The parasite-stress theory of sociality, the behavioral immune system, and human social and cognitive uniqueness. Evolutionary Behavioral Sciences, 8, 257-264.

Yzerbyt, V. Y., Leyens, J.-Ph., \& Bellour, F. (1995). The ingroup overexclusion effect: identity concerns in decisions about group membership. European Journal of Social Psychology, 25, 1-16.

Publisher's Note Springer Nature remains neutral with regard to jurisdictional claims in published maps and institutional affiliations. 